\title{
GW23-e2533 EFFECTS OF ENALAPRIL AND IRBESARTAN ON CAROTID ARTERY REMODELLING AND TGF-B1/SMADS PATHWAY IN RENOVASCULAR HYPERTENSIVE RATS
}

doi:10.1136/heartjnl-2012-302920a.14

Qian-Hui Shang, Jian-Ling Chen, Wei Hu, Chan Liu, Wan-Heng Mao, Qian-Hui Shang. Institute of Clinical Medicine, Department of cardiology of Affiliated Hospital, Zunyi Medical College, Zunyi Guizhou 563003, China

Objectives To investigate the effects of single-drug or combination therapy of enalapril and irbesartan on carotid artery remodelling and TGF- $\beta 1 /$ Smads signal pathway.

Methods Renovascular hypertensive rats (RHR) developed by 'twokidney and one-clip' method were treated respectively with distilled water (model group, $\mathrm{n}=6$ ), enalapril $(10 \mathrm{mg} /(\mathrm{kg} \mathrm{d}), \mathrm{n}=6)$, irbesartan $(50 \mathrm{mg} /(\mathrm{kg} / \mathrm{d}), \mathrm{n}=6)$ and enalapril plus irbesartan $(5 \mathrm{mg} /$ $\mathrm{kg} / \mathrm{d}+25 \mathrm{mg} /(\mathrm{kg} \mathrm{d}), \mathrm{n}=6)$ for 6 weeks. Six sham-operated rats were used as controls. Carotid artery morphology and structural changes were observed through $\mathrm{HE}$ staining, immunohistochemical staining and Masson staining. Media thickness (MT), lumen diameter (LD), media thickness and lumen diameter ratio (MT/LD) and collagen fibre area percentage of carotid arteries were measured. Moreover, the immunohistochemical staining was applied to detect the expression of alpha-smooth muscle actin ( $\alpha$-SMA), proliferating cell nuclear antigen (PCNA), TGF- $\beta 1, \mathrm{p}-\mathrm{Smad} 2 / 3$ and Smad7.

Results In the model group, the media thickness was significantly increased, and the volume of vascular smooth muscle cell (VSMC) increased and disarranged. MT, LD, MT/LD, $\alpha$-SMA, PCNA and collagen fibre area percentage of carotid arteries in the model group were higher than those in the sham-operated group $(p<0.01)$, and TGF- $\beta 1$ and $p$-smad2/3 were increased whereas Smad7 was decreased in the model group $(p<0.01)$. Single enalapril or irbesartan therapy decreased MT, MT/LD and the protein expression of TGF- $\beta 1$, p-Smad2/3, and increased the expression of Smad7. Combined enalapril and irbesartan treatment showed significant reductions in above experimental indices than single drug interventions (all $\mathrm{p}<0.05)$.

Conclusions The TGF $\beta 1 /$ Smads signalling pathway may be involved in carotid remodelling of RHR. Enalapril or irbesartan can attenuate carotid remodelling of RHR through regulating TGF- $\beta 1 /$ Smads pathway and both combination treatment seems to have interaction. 